\title{
Mini revisión: aplicación de líquidos iónicos en hidrólisis ácida de material lignocelulósico para la obtención de azúcares
}

\section{Mini Review: Application of Ionic Liquids in Acid Hydrolysis of Lignocellulosic Material to Obtain Sugars}

\author{
Angélica María Vele Salto ${ }^{1}$, Mónica Fernanda Abril González ${ }^{1}$, Teresita Silvana Zalamea Piedra ${ }^{1}$ y Verónica \\ Patricia Pinos Vélez ${ }^{1}$
}

\section{Resumen}

En las últimas décadas, la aplicación de líquidos iónicos (LI) como pretratamiento de la biomasa (BM) residual previo a la reacción de hidrólisis, ha demostrado ser un sistema eficiente para mejorar los rendimientos hacia la obtención de monosacáridos. Una mayor obtención de monosacáridos, se traduce en un mayor rendimiento en la producción de biocombustibles pudiendo volver a esta industria rentable. En este contexto, la presente revisión bibliográfica analiza las características fisicoquímicas, métodos de síntesis, condiciones de disolución con aplicación de variables, tales como: relación másica LI: BM, temperatura y tiempo. Además, se realiza un análisis comparativo de los resultados de procesos de hidrólisis ácida tradicional con respecto a los obtenidos con la aplicación de LI, aumentado en la mayoría de los casos sus rendimientos de reacción. Finalmente, se mencionan algunas técnicas de recuperación y reciclaje, que permitan reducir los costos del proceso, de manera que este sea económicamente rentable.

Palabras clave: biomasa, hidrólisis ácida, líquidos iónicos, material lignocelulósico

\begin{abstract}
In recent decades, the application of ionic liquids (IL) as pretreatment of residual biomass (BM) before the hydrolysis reaction has proven to be an efficient system to improve yields towards obtaining monosaccharides. A greater recovery of monosaccharides translates into a higher yield in the production of biofuels, which can make this industry profitable. In this context, this bibliographic review analyzes the physicochemical characteristics, synthesis methods, dissolution conditions with application of variables, such as: IL: BM weigh ratio, temperature and time. In addition, a comparative assessment of the results of traditional acid hydrolysis processes with respect to those obtained with the application of IL is carried out, increasing in most cases their reaction yields. Finally, some recovery and recycling techniques are mentioned, which allow to reduce the costs of the process, so that it is economically profitable.
\end{abstract}

Keywords: biomass, acid hydrolysis, ionic liquids, lignocellulosic material

Recepción: 11-nov-2020

Aceptación: 23-dic-2020

\footnotetext{
${ }^{1}$ Universidad de Cuenca, Departamento de Biociencias, IRCMA, Campus central, 12 de abril, Cuenca, Ecuador. Autor de correspondencia: monica.abrilg@ucuenca.edu.ec
} 


\section{Introducción}

Actualmente, el cambio de las fuentes de energía resultan ser un verdadero desafío para el área de la investigación, encaminada a la búsqueda de materia prima sostenible que permita la disminución del consumo de petróleo y a la vez aseguren la generación de biocombustibles y productos químicos con el menor impacto ambiental producido durante su ciclo de vida, lo que conlleva a la selección de materias primas renovables y el mejoramiento de las diversas etapas de transformación, necesarios para la obtención de los productos finales. El agotamiento de los recursos fósiles junto con las alarmas de calentamiento global ha llevado a una búsqueda intensificada de recursos alternativos para abastecer a la sociedad moderna [1]. Varios programas alrededor del mundo investigan los procesos de conversión de la biomasa, tales como: los residuos agrícolas, desechos forestales y cultivos energéticos. Para la obtención de biocombustibles se requiere al menos tres pasos principales: (i) pretratamiento para mejorar la liberación de los polímeros celulósicos de la estructura de la red de biomasa, (ii) hidrólisis de la biomasa celulósica pretratada en sus monosacáridos, como la glucosa, y (iii) fermentación o rehidratación de los azúcares monosacáridos a etanol u otros productos de biorefinería [2].

Desafortunadamente las características fisicoquímicas, estructurales y de composición de la biomasa la vuelven un material resistente al proceso de hidrólisis, siendo necesario su tratamiento con enzimas o ácidos bajo condiciones heterogéneas que permiten su descomposición en monosacáridos [3]. La biomasa usada está compuesta por materiales lignocelulósicos que se compone principalmente de celulosa, hemicelulosa y lignina [2]. Debido a la alta cristalinidad de la lignocelulosa en la biomasa natural, es difícil que un reactivo alcance la celulosa. Por lo tanto, su tratamiento previo es necesario para alterar la estructura cristalina de la celulosa, eliminar la hemicelulosa o eliminar o modificar la lignina, para aumentar la exposición del sustrato celulósico [4]. La clave para desbloquear la estructura recalcitrante de la lignocelulosa está en desarrollar un proceso efectivo de pretratamiento. Un nuevo método de pretratamiento prometedor para materiales lignocelulósicos es el uso de líquidos iónicos [5]. La capacidad única de los LI para disolver selectivamente los componentes de biomasa y la biomasa completa se ha demostrado tanto para los tipos de biomasa leñosa como herbácea. El pretratamiento de biomasa con LI ha sido aplicado con éxito para la producción de biocombustibles celulósicos [6]. Sin embargo, debido al alto costo de los LI, un aspecto a ser considerado para su aplicación a escala industrial, es la recuperación y reciclaje de los mismos [7].

\section{Biomasa lignocelulósica}

La biomasa lignocelulósica es el recurso renovable más abundante en la naturaleza, incluye: desechos forestales, residuos agrícolas, cultivos bioenergéticos, desechos industriales y desechos sólidos municipales [8]. Se compone principalmente de celulosa $(40-45 \%)$, hemicelulosa $(15-30 \%)$ y lignina $(16-33 \%)$ que se unen en una matriz compleja y entrelazada. La celulosa es un compuesto polimérico, formado por subunidades de D-glucosa, que se unen mediante enlaces glicosídicos $\beta-1,4$. Las cadenas de celulosa se acoplan mediante enlaces de hidrógeno y fuerzas de Van der Waals, lo que resulta en una alineación paralela y una estructura cristalina. La conversión de celulosa en glucosa ha sido un tema central por mucho tiempo. La celulosa se hidroliza para formar glucosa, que puede usarse como material de partida para diversos biocombustibles como el bioetanol y el butanol [4]. La hemicelulosa es el segundo polisacárido principal, es un componente amorfo, compuesto de diferentes unidades de monosacáridos, tales como: xilosa, arabinosa, galactosa y manosa [9], [10]. La hemicelulosa tiene un peso molecular más bajo que la celulosa y con cadenas laterales cortas que constan de diferentes polímeros de azúcares, fáciles de hidrolizar, mientras que la lignina es un polímero compuesto por varios grupos aromáticos [8], altamente ramificado, compuesto de guayacil propano, siringil propano y unidades de p-hidroxifenil propano, que une celulosa y hemicelulosa [2]. Para la producción de combustibles líquidos a partir de la fracción de carbohidratos de la biomasa lignocelulósica requiere su despolimerización en monosacáridos u otros 
productos de bajo peso molecular [11]. Para lo anterior, se debe reducir el contenido de lignina, disminuir la cristalinidad de la celulosa e incrementar el área superficial de contacto.

En la tabla 1 se muestran valores promedio de la composición y contenido de algunos materiales lignocelulósicos de interés para la producción de biocombustibles. Es importante mencionar que esta composición depende de las condiciones climáticas y del terreno en donde se hayan generado. Se puede observar que el bagazo y bambú presentan el mayor contenido de celulosa mientras que el ramón de olivo y la paja de maíz son los de menor cantidad. Por otra parte, la paja de trigo como el tallo de maíz son los únicos que tienen predominio de hemicelulosa sobre la celulosa.

Tabla 1. Contenido de celulosa, hemicelulosa y lignina de diferentes materiales lignocelulósicos

\begin{tabular}{lcccl}
\hline Biomasa & $\begin{array}{c}\text { Celulosa } \\
(\boldsymbol{\%})\end{array}$ & $\begin{array}{c}\text { Hemicelulosa } \\
(\boldsymbol{\%})\end{array}$ & Lignina (\%) & Referencia \\
\hline Alga Chlorella & 35.28 & 10.19 & - & (Zhou et al. 2012) \\
Pasto & 36.2 & 20.1 & 20.4 & $\begin{array}{l}\text { (Sun et al. 2013) } \\
\text { (van Spronsen }\end{array}$ \\
Madera de Pino & 39 & 26 & 35 & $\begin{array}{l}\text { et al. 2011) } \\
\text { (van Spronsen }\end{array}$ \\
Paja de trigo & 30 & 32 & 38 & et al. 2011) \\
Ramón de Olivo & 24.4 & 18.9 & 16.4 & (de la Rosa 2015) \\
Paja de cebada & 39.7 & 27.3 & 13.7 & (de la Rosa 2015) \\
Pasto (Miscanthus) & 42 & 25 & 28 & (Dee y Bell 2011) \\
Mazorca de maíz & 41.3 & 20 & 12.58 & (Chang et al. 2012) \\
Bagazo & 45.5 & 27 & 21.1 & (Jackson de Moraes \\
Madera de pino & 41.3 & 22.1 & 31.9 & (Sievers et al. 2009) \\
Loblolly & 28.3 & 16.4 & 23.8 & (Raj et al. 2015) \\
Paja de maíz & 39 & 42 & 7.3 & (Daud et al. 2014) \\
Tallo maíz & 38.1 & 19.9 & 14.1 & (Raj et al. 2015) \\
Paja de arroz & 32.5 & 23.3 & 28.2 & (Tarchoun, Trache, y \\
Klgapötke 2019) & & & & (Cao et al. 2014) \\
\hline Bambú & 45 & 26.1 & 23.1 &
\end{tabular}

Fuente: Los autores.

\section{Hidrólisis: Conceptos y tipos}

La despolimerización o hidrólisis del material lignocelulósico en productos intermedios más susceptibles a la transformación química o biológica es un pre-requisito o primer paso para la producción de biocombustibles o químicos de mayor valor [12-14].

La hidrólisis de la biomasa, es una etapa importante para la formación de azúcares, que constituyen la base para procesos posteriores de obtención de bioalcoholes y otros productos, de tal manera que mientras se alcance una mayor concentración en monosacáridos, mejor será el rendimiento hacia los productos finales de fermentación.

Los tipos de hidrólisis utilizados para la obtención de monosacáridos son:

- Hidrólisis ácida: Este proceso se realiza generalmente con soluciones diluidas o concentradas de ácidos a elevadas y bajas temperaturas respectivamente; ácidos como el clorhídrico $(\mathrm{HCl})$ y el sulfúrico $\left(\mathrm{H}_{2} \mathrm{SO}_{4}\right)$ se utilizan históricamente para estos procesos, sin embargo, existen experiencias con otros ácidos como son el ácido fluorhídrico (HF), nítrico $\left(\mathrm{HNO}_{3}\right)$, fosfórico $\left(\mathrm{H}_{3} \mathrm{PO}_{4}\right)$ y ácidos orgánicos, entre otros. Las soluciones ácidas concentradas pueden disolver la celulosa utilizando bajas temperaturas, lo que energéticamente es más favorable que utilizar ácidos diluidos a elevadas temperaturas, sin embargo, esto conducen a la producción de elevadas cantidades de residuos de neutralización, que son difíciles de reciclar y muy corrosivos. Tratamientos ácidos severos también conducen a la degradación de azúcares a furanos como el furfural y el 5-hidroximetilfurfural (HMF), ácidos carboxílicos y sustancias húmicas insolubles, tratamientos ácidos menos severos previenen la degradación de azúcares, sin embargo, son menos eficientes $[10,15]$.

- Hidrólisis alcalina: Este proceso elimina la lignina a través de escisión de los enlaces de éter y solubiliza la hemicelulosa a través de la saponificación de los enlaces éster intermoleculares entre hemicelulosa y lignina, aumentando la porosidad y la accesibilidad general a la celulosa. Los productos químicos de pretratamiento alcalino incluyen: $\mathrm{NaOH}, \mathrm{Ca}(\mathrm{OH})_{2}$ y $\mathrm{NH}_{3}$ y pueden usarse en combinación con agentes oxidativos. Una de las limitaciones asociadas al tratamiento alcalino es la conversión en sales irrecuperables o la incorporación de estas en la biomasa, que perjudica los tratamientos posteriores $[10,15]$. 
- Hidrólisis enzimática: se realiza mediante la aplicación de microorganismos y enzimas para la descomposición del material lignocelulósico en monosacáridos. La hidrólisis enzimática de materiales lignocelulósicos puede ser influenciada no solo por la efectividad de las enzimas sino también por las características físicas, químicas y morfológicas de los materiales lignocelulósicos. La mayoría de los factores que afectan la hidrólisis enzimática pueden dividirse en dos grupos: los relacionados a las enzimas, o a los sustratos. Los relacionados con las enzimas, se centra principalmente en mejorar la actividad enzimática, incluyendo la inhibición del producto final, estabilidad térmica, sinergia y adsorción. Los factores relacionados con el sustrato, se centran principalmente en mejorar la accesibilidad de las enzimas a la celulosa (Sun et al. 2016). Sin embargo, el principal inconveniente de este proceso se debe al bajo rendimiento de reacción y al elevado costo de las enzimas [13].

A nivel industrial el proceso más importante es la hidrólisis con ácidos minerales concentrados o diluidos, predominando el ácido sulfúrico, debido a que es el proceso más simple y presenta su ventaja frente a los demás tipos de hidrólisis que es su alta velocidad. El tratamiento con ácido sulfúrico diluido ha sido ampliamente estudiado debido a que es considerado, económico, conveniente y efectivo para biomasa lignocelulósica [16]. A lo largo de los años, se han ido efectuando una serie de tratamientos con el objetivo de obtener un mejor rendimiento en la reacción de hidrólisis ácida, experimentado la influencia de diferentes variables, tales como: tiempo, temperatura y concentración de ácido. En la figura 1 se representa el proceso de hidrólisis ácida en biomasa.

En la tabla 2 se presentan los resultados obtenidos de la reacción de hidrólisis ácida en diferentes tipos de biomasas.

Se puede observar en la tabla 2 que el ácido más utilizado es el ácido sulfúrico en bajas concentraciones, a excepción del estudio realizado por Wijaya y colaboradores [27] que utilizan una concentración

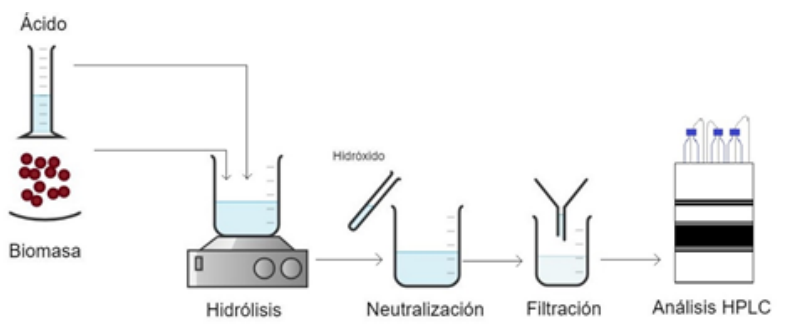

Figura 1. Proceso de hidrólisis ácida en biomasa.

Tabla 2. Resultados de la reacción de hidrólisis ácida de diferentes tipos de biomasa

\begin{tabular}{|c|c|c|c|c|c|c|}
\hline Biomasa & Ácido & $\begin{array}{c}\text { Concentración } \\
\% \mathrm{v} / \mathrm{v}\end{array}$ & $\begin{array}{c}\text { Temperatura } \\
\left({ }^{\circ} \mathrm{C}\right)\end{array}$ & $\begin{array}{c}\text { Tiempo } \\
\text { (min) }\end{array}$ & Rendimiento & eferencia \\
\hline $\begin{array}{l}\text { Alga } \\
\text { Chlorella }\end{array}$ & $\mathrm{H}_{2} \mathrm{SO}_{4}$ & $1 \%$ & 121 & 20 & $\begin{array}{l}90.4 \% \\
\text { glucosa }\end{array}$ & [18] \\
\hline Pasto & $\mathrm{H}_{2} \mathrm{SO}_{4}$ & $1 \%$ & 140 & 60 & $\begin{array}{c}14.3 \% \\
\text { glucosa }\end{array}$ & [19] \\
\hline $\begin{array}{l}\text { Madera de } \\
\text { Pino }\end{array}$ & $\mathrm{H}_{3} \mathrm{PO}_{4}$ & $4.49 \%$ & 106.7 & 274.2 & $\begin{array}{c}90.85 \% \\
\text { xilosa }\end{array}$ & [20] \\
\hline Paja de trigo & $\mathrm{H}_{2} \mathrm{SO}_{4}$ & $5 \%$ & 130 & 180 & $\begin{array}{c}37 \% \\
\text { glucosa }\end{array}$ & [21] \\
\hline $\begin{array}{l}\text { Ramón de } \\
\text { Olivo }\end{array}$ & $\mathrm{H}_{2} \mathrm{SO}_{4}$ & $4 \%$ & 120 & 30 & $\begin{array}{l}6.20 \% \\
\text { glucosa }\end{array}$ & [22] \\
\hline $\begin{array}{l}\text { Paja de } \\
\text { cebada }\end{array}$ & $\mathrm{H}_{2} \mathrm{SO}_{4}$ & $1 \%$ & 140 & 120 & $\begin{array}{c}15 \% \\
\text { glucosa }\end{array}$ & [22] \\
\hline $\begin{array}{l}\text { Pasto } \\
\text { (Miscanthus) }\end{array}$ & $\mathrm{H}_{2} \mathrm{SO}_{4}$ & $0.25 \%$ & 200 & 40 & $\begin{array}{c}7.07 \% \\
\text { glucosa }\end{array}$ & [23] \\
\hline $\begin{array}{l}\text { Mazorca de } \\
\text { maíz }\end{array}$ & $\mathrm{HCl}$ & $5.8 \%$ & 108 & 80 & $\begin{array}{c}1.2 \mathrm{~g} \\
\text { glucosa/L } \\
\text { de solución } \\
\text { hidrolizada }\end{array}$ & [24] \\
\hline $\begin{array}{l}\text { Mazorca de } \\
\text { maíz }\end{array}$ & $\mathrm{H}_{2} \mathrm{SO}_{4}$ & $2 \%$ & 160 & 30 & $\begin{array}{c}66.8 \% \\
\text { glucosa } \\
(18,4 \mathrm{~g} / \mathrm{L})\end{array}$ & [25] \\
\hline $\begin{array}{l}\text { Bagazo de } \\
\text { caña de } \\
\text { azúcar }\end{array}$ & $\mathrm{H}_{2} \mathrm{SO}_{4}$ & $2 \%$ & 155 & 10 & $\begin{array}{c}70 \% \\
\text { glucosa }\end{array}$ & [26] \\
\hline $\begin{array}{l}\text { Madera de } \\
\text { pino Loblolly }\end{array}$ & $\mathrm{H}_{2} \mathrm{SO}_{4}$ & $80 \%$ & 80 & 60 & $\begin{array}{l}29.83 \% \\
\text { glucosa }\end{array}$ & [27] \\
\hline Paja de maíz & $\mathrm{H}_{2} \mathrm{SO}_{4}$ & $2 \%$ & 120 & 43 & $\begin{array}{c}8.4 \% \\
\text { glucosa }\end{array}$ & [28] \\
\hline Tallo maíz & $\mathrm{H}_{2} \mathrm{SO}_{4}$ & $2 \%$ & 160 & 30 & $\begin{array}{c}64.1 \% \\
\text { glucosa }\end{array}$ & [25] \\
\hline Paja de arroz & $\mathrm{H}_{2} \mathrm{SO}_{4}$ & $0.8 \%$ & 190 & 4 & $\begin{array}{c}8.52 \% \\
\text { glucosa }\end{array}$ & [29] \\
\hline Alga Parda & $\mathrm{H}_{2} \mathrm{SO}_{4}$ & $1 \%$ & 121 & 15 & $\begin{array}{l}43.4 \% \\
\text { glucosa }\end{array}$ & [30] \\
\hline Bambú & $\mathrm{H}_{2} \mathrm{SO}_{4}$ & $1.2 \%$ & 140 & 90 & $\begin{array}{c}3.5 \% \\
\text { glucosa }\end{array}$ & [31] \\
\hline
\end{tabular}

de $80 \%$ para la madera de pino Loblolly y obtienen un rendimiento de reacción a glucosa de $29.83 \%$. Los rangos de temperatura y tiempos de reacción fueron amplios de 80 a $200{ }^{\circ} \mathrm{C}$ y 4 a $274.2 \mathrm{~min}$, respectivamente. Por otra parte, la influencia de la concentración del ácido, temperatura y tiempo de reacción es notoria, Yan y colaboradores [19] trataron el pasto con $\mathrm{H}_{2} \mathrm{SO}_{4}$ al $1 \%$, por 60 min a una temperatura de $140{ }^{\circ} \mathrm{C}$, obteniendo un $14,3 \%$ 
de glucosa, Świątek y colaboradores [23], llevaron a cabo la reacción de hidrólisis a una concentración del $0.25 \%$, con una reducción del tiempo y aumento de temperatura, alcanzando un $7.07 \%$ de glucosa, valor inferior al obtenido por Yan [19]. Otro tipo de biomasa de interés son las algas, Ho y colaboradores[18] desarrollaron una reacción de hidrólisis para el alga chlorella y obtuvieron un rendimiento de reacción para glucosa de $90.4 \%$, utilizando $\mathrm{H}_{2} \mathrm{SO}_{4}$ al $1 \%$ por $20 \mathrm{~min}$ a una temperatura de $121{ }^{\circ} \mathrm{C}$; También Greetham y colaboradores [30], llevaron a cabo la reacción de alga parda y obtuvieron un rendimiento para glucosa de $43.4 \%$ utilizando el mismo ácido en la misma concentración y temperatura, variando únicamente el tiempo de reacción que es de $15 \mathrm{~min}$. En este caso la disminución en el rendimiento puede verse afectado por el cambio en la especie de alga y/o por el tiempo de reacción. Además, los residuos de la planta de maíz, que dan mejores resultados en la conversión a azúcares, son el tallo y la mazorca obteniendo hasta un $64.1 \%$ y $66.8 \%$ de glucosa, respectivamente.

Varios estudios han demostrado que, para extraer los monosacáridos del material lignocelulósico, la celulosa y hemicelulosa deben liberarse de la lignina, y luego ser hidrolizados. Esta conversión eficiente es un desafío para la producción económica de biocombustibles, aunque la hemicelulosa se hidroliza fácilmente se necesita un pretratamiento que permita eliminar la lignina, reducir la cristalinidad de la celulosa y aumentar la porosidad de la celulosa. Por lo que para mejorar los rendimientos de hidrólisis se han propuesto pretratamientos que permitan lograr estas condiciones. Los pretratamientos a la hidrólisis más comunes son ácido diluido, agua caliente y la aplicación de amoniaco acuoso para la expansión de las fibras; sin embargo, estas técnicas no son amigables con el medio ambiente y resultan en la pérdida de valiosas fracciones de carbohidratos de la biomasa [32]. Estudios recientes han demostrado que los líquidos iónicos son una gran promesa para el pre-tratamiento de la celulosa [33], debido a que facilitan de una forma extraordinaria la hidrólisis de los biopolímeros ya que eliminan las barreras de protección estructurales de los centros de hidrólisis, permitiendo que la reacción se lleve a temperaturas incluso por debajo de $100{ }^{\circ} \mathrm{C}$ [22], resolviendo de esta manera la principal desventaja del proceso de hidrólisis diluida que requería elevados requerimientos energéticos.

\subsection{Hidrólisis ácida con pretratamiento con líquidos iónicos}

Los líquidos iónicos (LI) son relativamente la nueva clase de materiales amigables con el medio ambiente, siendo considerados como los candidatos ideales para un amplio rango de aplicaciones, incluyendo: la adsorción de gas, solventes de extracción, almacenamiento de energía, electroquímica, síntesis orgánica, etc. Sus propiedades varían con el grupo sustitutivo sobre el catión o con la combinación del anión, ampliando su rango de aplicación en el campo catalítico. Desde las primeras investigaciones han sido considerados cruciales para los procesos de hidrólisis [28].

Los LI son sales orgánicas compuestas por pares de cationes y aniones que tienen estructuras asimétricas como se observa en la tabla 3 , lo que explica sus bajos puntos de fusión $\left(\leq 100{ }^{\circ} \mathrm{C}\right)$. Se denominan "designer solvents" ya que su miscibilidad, punto de fusión, viscosidad, basicidad de enlace de hidrógeno y polaridad se pueden adaptar ajustando los componentes de cationes y/o aniones [7], [34]. Generalmente, tienen alta estabilidad térmica y química, amplia región líquida, presión de vapor insignificante, reciclabilidad y no inflamabilidad, considerándose solventes amigables con el medio ambiente y eficientes para el procesamiento de la biomasa. Lo más crítico es que tienen un alto poder de solvatación, lo que les permite disolver muchas sustancias con diferentes polaridades y estructuras, incluida la biomasa que contiene: celulosa, hemicelulosa, lignina, quitina y seda. Pueden reducir significativamente la cristalinidad de la biomasa al alterar sus estructuras moleculares y permitir que los agentes de hidrólisis penetren fácilmente en la matriz. Además, el pretratamiento de la biomasa se lleva a cabo en condiciones relativamente leves lo que podría aliviar la formación de inhibidores y reducir los requerimientos de energía [34].

Debido a estas características, los LI son aceptados como solventes prometedores para romper los enlaces de hidrógeno inter-intramolecular de la celu- 
losa nativa de estructura cristalina, rompiendo los principales enlaces químicos en polímeros matriciales de diversas materias primas de biomasa. Se conoce que el pretratamiento con líquidos iónicos disminuye la cristalinidad de la celulosa, elimina parcialmente la lignina y hemicelulosa y aumenta el área de superficie específica, mejorando así la digestibilidad y fermentabilidad de azúcares a bajas temperaturas en condiciones ambientales. Algunos resultados muestran que la temperatura y el tiempo de pretratamiento son cruciales y afectan intensamente el rendimiento de la sacarificación. Por lo tanto, la selección de las condiciones adecuadas de pretratamiento es necesario para aumentar la eficiencia y minimizar el consumo de energía del proceso [35]. Los LI son los solventes más adecuados para varios tipos de reacciones, tales como: polimerización, alquilación, hidrólisis ácida, despolimerización, etc. [7]. Su uso como solvente es una alternativa atractiva para el pretratamiento de la biomasa lignocelulósica (Sun et al. 2013).

\subsection{Síntesis de LI}

Los LI se dividen en dos categorías principales: LI simples (formadas por un anión y un catión) y líquidos iónicos binarios (sales en donde está involucrado el equilibrio químico). La síntesis de LI puede ser descrito en dos pasos: 1. Formación del catión deseado: Este puede ser sintetizado por la protonación de la amina por un ácido o mediante reacciones de cuaternización de amina con un haloalcano y se procede a calentar la muestra. 2. Intercambio de anión: Estas reacciones pueden desarrollarse mediante el tratamiento de sales de haluro con ácido de Lewis para formar Lewis "acid-based ionic liquids" o por metátesis aniónica [36]. Sin embargo, en la mayoría de los casos es necesario un único paso, este es para la síntesis de líquidos iónicos, como es el caso de: Cloruro de 1-butil-3-metilimidazolio, nitrato de etil amonio, etc. Los líquidos iónicos basados en resinas de intercambio iónico y "brönsted acidic" también se sintetizan a través de la reacción de intercambio de aniones. Las sales basadas en $\mathrm{AlCl}_{3}$ que actúan como "Lewis acidic Lis" se estudian ampliamente para algunas aplicaciones; esta es una reacción de adición muy simple en la que el ácido de Lewis o los haluros metálicos son añadidos a las sales de haluro dando como resultado la formación de LIs ácidos con una especie de haluro adicional [7]. Además de estos métodos convencionales, también se han desarrollado una variedad de metodologías para sintetizar series de LI mediante irradiación de microondas (MW), sonicación, LI macrocíclicas, apertura de anillo, neutralización ácido-base, éteres corona, ultrasonido de potencia (HPU) y muchos más. [7].

\subsection{Toxicidad, biodegradabilidad y renovabilidad}

Si bien las investigaciones iniciales se centraron en los LI basados en cloruro, se apoyó la transición hacia compuestos con toxicidades más bajas. Los que contienen cloruro poseen toxicidades y propiedades corrosivas que probablemente no serían adecuadas para la mayoría de las operaciones de biorefinación. Los LI biodegradables son deseables para minimizar el impacto ambiental de los productos químicos y para que cumplan con su facturación como solventes verdes, especialmente con el control cada vez más estricto de las regulaciones ambientales sobre el uso de solventes industriales [6]. Sin embargo, existen importantes barreras técnicas, económicas y ambientales para la comercialización a gran escala de LI en biorefinerías. Para superar estas barreras, se recomienda que se realicen investigaciones para el desarrollo de LI que puede combinar la eficacia para el pretratamiento de biomasa con la capacidad de usarse en condiciones hidratadas (lo que también reduce su viscosidad). Los LI presentan las siguientes ventajas: son térmicamente estables e inertes en condiciones de pretratamiento; pueden ser reciclados y reutilizados; no son tóxicos (para la vida humana y los organismos microbiológicos); y son de bajo costo (en comparación con los solventes industriales actuales). Además, para que los LI logren una identidad como tecnologías sostenibles en un contexto de biorefinería, sería apropiado que se fabriquen con los mismos recursos renovables que pre-tratan para la producción de productos químicos y materiales renovables [6]. 
Tabla 3. Líquidos iónicos para decristalización más usados

\begin{tabular}{lcccccc}
\hline Nombre & Abreviación & CAS & masa molar g/mol Estructura \\
Cloruro de 1-etil-3-metilimidazolio & {$[\mathrm{Emim}][\mathrm{Cl}]$} & $65039-09-0$ & 146.62 & \\
Cloruro de 1-butil-3-metilimidazolio & {$[\mathrm{Bmim}][\mathrm{Cl}]$} & $79917-90-1$ & & \\
\hline
\end{tabular}

$\begin{array}{llll}\text { Bromuro de 1-etil-3-metilimidazolio } \quad[\text { Emim }] \mathrm{Br} & 65039-08-9 & 191.07\end{array}$

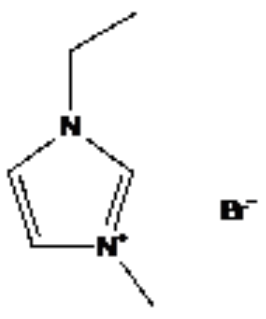

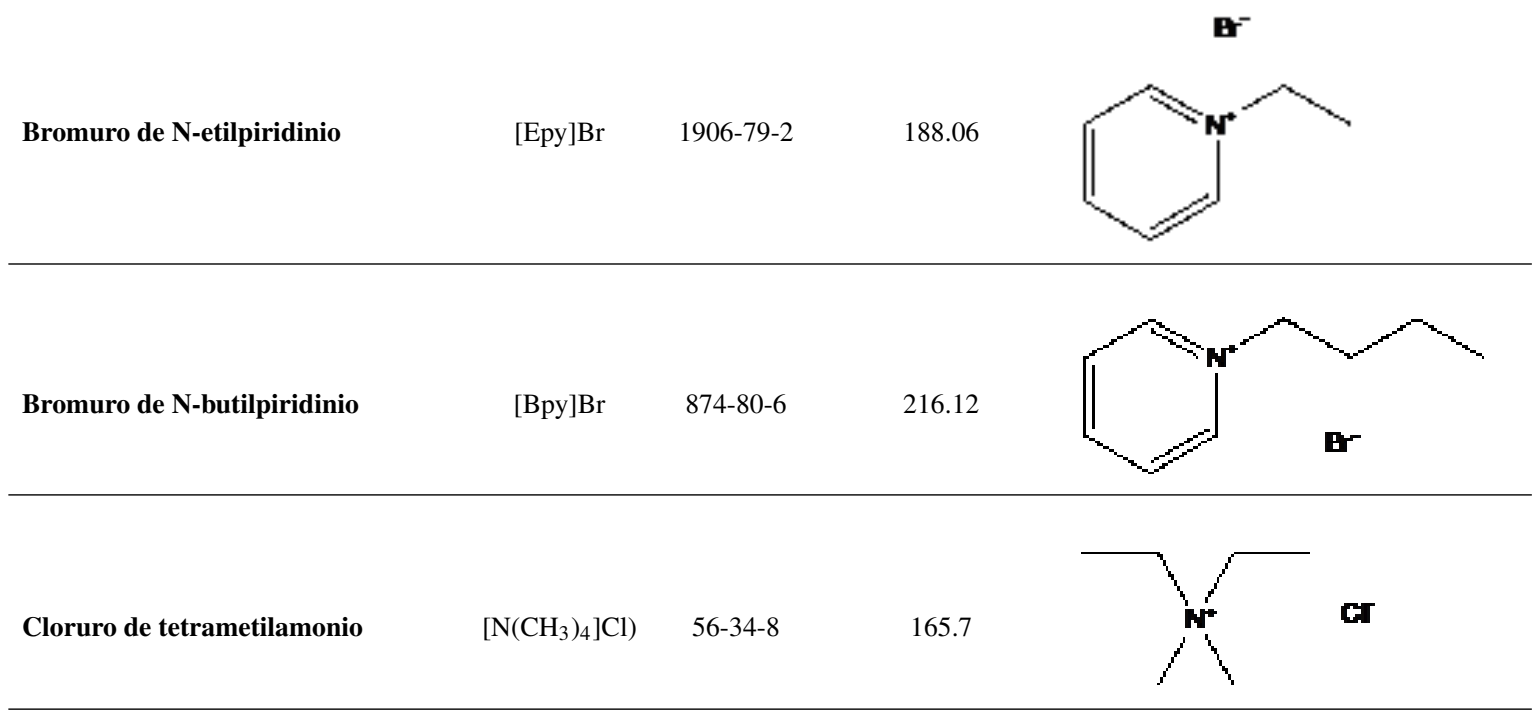

Acetato de 1-butil-3-metilimidazolio [Bmim]OAc 284049-75-8

198.26

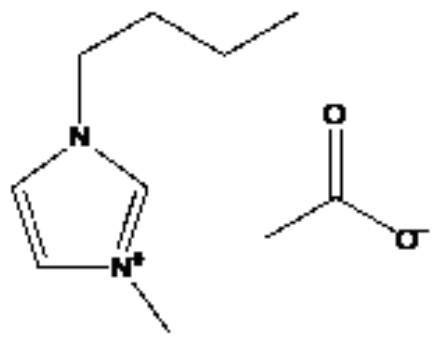


Actualmente, se realiza la aplicación de LI como método de pretratamiento para aumentar los rendimientos alcanzados al aplicar las técnicas tradicionales de hidrólisis. En la figura 2 se observa el esquema de los principales pasos requeridos en este proceso de pretratamiento.

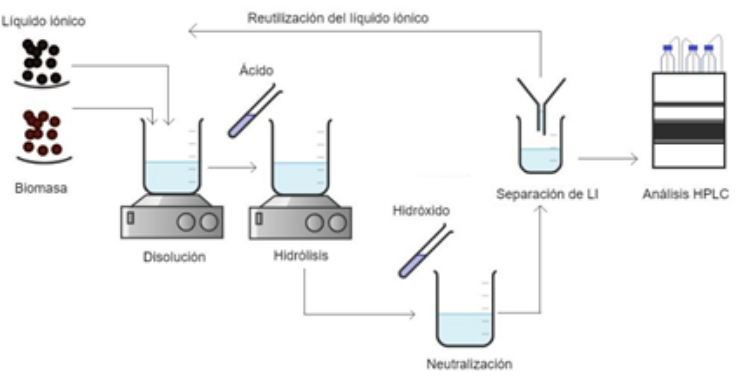

Figura 2. Pretratamiento de biomasa con líquidos iónicos.

En la tabla 4 se realiza un resumen de los resultados obtenidos en la reacción de hidrólisis ácida aplicando un pre-tratamiento con LI en diferentes biomasas que han sido de interés para la obtención de biocombustibles.

En la tabla 4 se observa que, para el tratamiento de los diferentes tipos de biomasa analizados en este estudio, los líquidos iónicos más comunes son: [Emim][Cl]; [BMIM] Cl y [C4mim]Cl su estructura se puede observar en la tabla 3 y los ácidos aplicados como catalizadores en la reacción de hidrólisis son: $\mathrm{H}_{2} \mathrm{SO}_{4}$ y HCl .

En la mayoría de los casos, la aplicación de líquidos iónicos como pretratamiento a la hidrólisis, mejora los rendimientos de reacción hacia monosacáridos; por ejemplo, en el pasto según Świątek [23] obtiene un rendimiento a glucosa de $7.07 \%$ sin pretratamiento, mientras que Sun, para la misma biomasa alcanza 53\% de glucosa, con un pretratamiento con LI ([Bmim][Cl]) [23]. Zhou realizó un pretratamiento aplicando $[\mathrm{Emim}][\mathrm{Cl}]$ y utilizó $\mathrm{HCl}$ para el proceso de hidrólisis, logrando mejorar el rendimiento de reacción para glucosa a $90.4 \%$ [37], por otra parte Malihan, Nisola y Chung realizaron el pretratamiento para el alga parda con [BMIM]Cl y utilizaron $\mathrm{HCl}$ para el proceso de hidrólisis, mejorando de igual manera el rendimiento de reacción a glucosa a 66\% [40]. Para los dos tipos de algas se puede observar que la aplicación del pretratamiento con líquidos iónicos mejora el rendimiento de reacción. Una excepción a esta mejora es el bagazo de caña en el cual disminuye el rendimiento; en este caso se debería profundizar el estudio de las variables que influyen en la reacción, incluyendo la especie de biomasa analizada y su composición estructural.

El tipo de líquido iónico aplicado en el pretratamiento de biomasa también influye en el rendimiento de reacción como es el caso de la paja de trigo. Estudios realizados para la reacción de hidrólisis sin la aplicación de un pretratamiento obtiene un rendimiento a glucosa de $37 \%$ utilizando como catalizador de reacción $\mathrm{H}_{2} \mathrm{SO}_{4}$. Comparando estos resultados con los obtenidos con la aplicación del pretratamiento con líquidos iónicos en el caso de la paja de trigo que utiliza [Bmim] $\mathrm{Cl}$ y $\mathrm{H}_{2} \mathrm{SO}_{4}$ como ácido para el proceso de hidrólisis, se obtiene un rendimiento de $48 \%$, superior al obtenido sin pretratamiento y de igual manera, superior al obtenido por de La Rosa [22] que utiliza [EMIM]Cl y HCl..

Otro de los factores que tiene influencia sobre los resultados de la reacción es la relación líquido iónico-biomasa, como se da en la mazorca de maíz, al disolver $26.7 \mathrm{mg}$ de biomasa en $502 \mathrm{mg}$ de (EMIM)Cl, a $105^{\circ} \mathrm{C}$, durante $6 \mathrm{~h}$, con la posterior reacción de hidrólisis ácida con $\mathrm{HCl}$ al $1.66 \mathrm{M}$, a $105^{\circ} \mathrm{C}$ durante $5.5 \mathrm{~h}$, obteniendo un $70 \%$ de glucosa. [39]. Al modificar la relación másica LI:BM a 10:1, y aumentando la concentración del $\mathrm{HCl}$ al $20 \%$, se favorece el rendimiento a glucosa al 78\% [12].

\subsection{Recuperación de Líquidos Iónicos}

Debido al alto costo actual de los líquidos iónicos, su recuperación y reciclaje es requerido para su uso en el pretratamiento de la biomasa. Los LI tienen la propiedad de disolver la celulosa en condiciones moderadas y pueden recuperarse en casi un $100 \%$ de pureza original. Además de aumentar la carga de biomasa en los LI, su recuperación y reciclaje representa un medio importante para ahorrar costos en el proceso, el mismo que se ve facilitado por sus volatilidades insignificantes en condiciones ambientales $[4,6,7]$. 
Tabla 4. Resultados de hidrólisis ácida asistida con líquidos iónicos en diferentes tipos de biomasa. Condiciones de pretratamiento y condiciones de hidrólisis

\begin{tabular}{|c|c|c|c|c|c|c|}
\hline Biomasa & Líquido iónico & Relación másica LI/BM & Condiciones de pretratamiento & Condiciones de hidrólisis & Rendimiento $\%$ & Referencia \\
\hline Alga Chlorella & {$[\mathrm{Emim}][\mathrm{Cl}]$} & $\mathrm{LI}=310 \mathrm{mg} \mathrm{BM}=15.2 \mathrm{mg}$ & $105^{\circ} \mathrm{C}$ y $3 \mathrm{~h}$ & $\mathrm{HCl}, 105^{\circ} \mathrm{C}$ y $3 \mathrm{~h}$ & $94 \%$ glucosa & [37] \\
\hline Pasto & {$[\mathrm{Bmim}][\mathrm{Cl}]$} & $\mathrm{LI}=10 \mathrm{~g} \mathrm{BM}=0.5 \mathrm{~g}$ & $160^{\circ} \mathrm{C}$ y $1.5 \mathrm{~h}$ & $\mathrm{HCl}, 105^{\circ} \mathrm{C}$ y $2.5 \mathrm{~h}$ & $53 \%$ glucosa & [24] \\
\hline Madera de Pino & {$[\mathrm{Emim}][\mathrm{Cl}]$} & $\mathrm{LI}=10 \mathrm{~g} \mathrm{BM}=0.5 \mathrm{~g}$ & $99.85^{\circ} \mathrm{C}$ y $24 \mathrm{~h}$ & $2 \mathrm{ml}$ de ácido acético, $124.85^{\circ} \mathrm{C}$ y $5 \mathrm{~h}$ & $3.2 \%$ furfural & [1] \\
\hline Paja de trigo & {$[\mathrm{Bmim}] \mathrm{Cl}$} & $\mathrm{LI}=$ & $80^{\circ} \mathrm{C}$ y $0.5 \mathrm{~h}$ & $\mathrm{H}_{2} \mathrm{SO}_{4}$ al $2 \%, 80^{\circ} \mathrm{C}$ y $0.5 \mathrm{~h}$ & $48 \%$ glucosa & [2] \\
\hline Paja de trigo & {$[\mathrm{Emim}][\mathrm{Cl}]$} & $\mathrm{LI}=10 \mathrm{~g} \mathrm{BM}=0.5 \mathrm{~g}$ & $100^{\circ} \mathrm{C}$ y $24 \mathrm{~h}$ & Ácido Acético $2 \mathrm{ml}, 125^{\circ} \mathrm{C}$ y $5 \mathrm{~h}$ & $4.5 \%$ furfural & [1] \\
\hline Paja de trigo & [EMIM]Cl & $\mathrm{LI}=9.5 \mathrm{~g} \mathrm{BM}=0.5 \mathrm{~g}$ & $135^{\circ} \mathrm{C}$ y $2.5 \mathrm{~h}$ & $\mathrm{HCl} 1.66 \mathrm{M}, 105^{\circ} \mathrm{C}$ y $3 \mathrm{~h}$ & $38 \%$ glucosa & [22] \\
\hline Ramón de Olivo & {$[\mathrm{EMIM}] \mathrm{Cl}$} & $\mathrm{LI}=9.5 \mathrm{~g} \mathrm{BM}=0.5 \mathrm{~g}$ & $135^{\circ} \mathrm{C}$ y $2.5 \mathrm{~h}$ & $\mathrm{HCl} 1.66 \mathrm{M}, 105^{\circ} \mathrm{C}$ y $3 \mathrm{~h}$ & $33 \%$ azúcares reductores totales & [22] \\
\hline Paja de cebada & [EMIM]Cl & $\mathrm{LI}=9.5 \mathrm{~g} \mathrm{BM}=0.5 \mathrm{~g}$ & $135^{\circ} \mathrm{C}$ y $2.5 \mathrm{~h}$ & $\mathrm{HCl} 1.66 \mathrm{M}, 105^{\circ} \mathrm{C}$ y $3 \mathrm{~h}$ & $39 \%$ glucosa & [22] \\
\hline Paja de cebada & [EMIM]Cl & $\mathrm{LI}=9.5 \mathrm{~g} \mathrm{BM}=0.5 \mathrm{~g}$ & $135^{\circ} \mathrm{C}$ y $1.5 \mathrm{~h}$ & $\mathrm{H}_{2} \mathrm{SO}_{4} 0.2 \mathrm{M}, 140^{\circ} \mathrm{C}$ y $2 \mathrm{~h}$ & $2.3 \mathrm{~g} / \mathrm{L}$ azúcares reductores totales & [22] \\
\hline Pasto (Miscanthus) & [EMIM]Cl & $\mathrm{LI}=500 \pm 20 \mathrm{mg} \mathrm{BM}=0.5 \mathrm{~g}$ & $104.85^{\circ} \mathrm{C}$ y $6 \mathrm{~h}$ & $\mathrm{H}_{2} \mathrm{SO}_{4} 1.66 \mathrm{M}, 99.85^{\circ} \mathrm{C}$ y $120 \mathrm{~min}$ & $63 \%$ glucosa & [38] \\
\hline Mazorca de maíz & [EMIM]Cl & $\mathrm{LI}=502 \mathrm{mg} \mathrm{BM}=26.7 \mathrm{mg}$ & $105^{\circ} \mathrm{C}$ y $6 \mathrm{~h}$ & $\mathrm{HCl} 1.66 \mathrm{M}, 105^{\circ} \mathrm{C}$ y $5.5 \mathrm{~h}$ & $70 \%$ glucosa & [39] \\
\hline Mazorca de maíz & [EMIM]Cl & LI:BM 10:1 & $105^{\circ} \mathrm{C}$ & $\mathrm{HCl} 20 \%$ & $78 \%$ glucosa & [12] \\
\hline Bagazo & {$[\mathrm{Bmim}] \mathrm{Cl}$} & & $80^{\circ} \mathrm{C} 0.5 \mathrm{~h}$ & $\mathrm{H}_{2} \mathrm{SO}_{4} 2 \%, 160^{\circ} \mathrm{C}$ y $1.5 \mathrm{~h}$ & $33 \%$ glucosa & [2] \\
\hline Madera de Pino & $\mathrm{C} 4 \mathrm{mimCl}$ & $\mathrm{LI}=4 \mathrm{~g} \mathrm{BM}=0.2 \mathrm{~g}$ & $100^{\circ} \mathrm{C} 0.5 \mathrm{~h}$ & $\mathrm{HCl}$ & $81 \%$ azúcares reductores totales & [13] \\
\hline Paja de maíz & {$[\mathrm{BMIM}] \mathrm{Cl}$} & $\mathrm{LI}(\mathrm{mg})=0.08 \mathrm{p} / \mathrm{p}$ de $\mathrm{BM}$ & $150^{\circ} \mathrm{C} 2 \mathrm{~h}$ & $\mathrm{H}_{2} \mathrm{SO}_{4} 0.5 \mathrm{p} / \mathrm{p}$ de BM, $150^{\circ} \mathrm{C}$ y $0.5 \mathrm{~h}$ & $35.5 \%$ azúcares reductores totales & [4] \\
\hline Tallo maíz & $\mathrm{C} 4 \mathrm{mimCl}$ & $\mathrm{LI}=4 \mathrm{~g} \mathrm{BM}=0.2 \mathrm{~g}$ & $100^{\circ} \mathrm{C}$ y $0.5 \mathrm{~h}$ & $\mathrm{HCl}$ & $\begin{array}{c}66 \% \text { azúcares reductores totales } \\
48 \% \text { glucosa }\end{array}$ & [13] \\
\hline Paja de arroz & $\mathrm{C} 4 \mathrm{mimCl}$ & $\mathrm{LI}=4 \mathrm{~g} \mathrm{BM}=0.2 \mathrm{~g}$ & $100^{\circ} \mathrm{C}$ y $0.5 \mathrm{~h}$ & $\mathrm{HCl}$ & $74 \%$ azúcares reductores totales & [13] \\
\hline Alga Parda & {$[\mathrm{BMIM}] \mathrm{Cl}$} & Biomasa en [BMIM] Cl al 5\% & Temperatura Ambiente y $24 \mathrm{~h}$ & $\mathrm{HCl} 30 \%, 120^{\circ} \mathrm{C}$ y $45 \mathrm{~min}$ & $66 \%$ glucosa & [40] \\
\hline Bambú & {$[\mathrm{BMIM}] \mathrm{Cl}$} & $\mathrm{LI}=4 \mathrm{~g} \mathrm{BM}=0.1 \mathrm{~g}$ & No establecida & $\mathrm{H}_{2} \mathrm{SO}_{4} 5 \%, 180^{\circ} \mathrm{C}$ y $2 \mathrm{~h}$ & $72 \%$ azúcares reductores totales & [41] \\
\hline
\end{tabular}

En un proceso típico de pretratamiento con líquidos iónicos, se mezcla la biomasa molida y seca con el líquido iónico y se calienta hasta que una parte o toda la biomasa se haya disuelto. La mayor parte del contenido celulósico de la biomasa se puede recuperar de la solución mediante la adición de un agente precipitante que generalmente es agua o un alcohol, este precipita la celulosa (fracción rica en glucano) y esta se puede recuperar mediante filtración o centrifugación. Con la adición de agua generalmente se observa la formación de una fase gel, lo que dificulta la separación de la celulosa. Si se emplea una solución agua:acetona 1:1 v/v se evita la formación del gel. Otra alternativa para su recuperación se da debido a su capacidad para formar un sistema bifásico con la adición de una solución acuosa que contenga un anión kosmotrópico, tal como: fosfato, carbonato o sulfato (Shill et al. 2011; Sun et al. 2013).

Gao, Bandara y Griffin efectuaron un estudio en el pretratamiento de biomasa lignocelulósica que utilizan sales inorgánicas como adyuvantes de mezclas agua-líquido iónico. Después del pretratamiento y regeneración de la biomasa, se elimina el sobrenadante que contiene principalmente LI y sales inorgánicas. Luego, se adiciona $40 \mathrm{~g}$ de sal inorgánica a la solución. La mezcla obtenida se somete a vibración y se deja reposar a $25{ }^{\circ} \mathrm{C}$ durante la noche, formando un sistema bifásico acuoso de una fase rica en LI. Después se separan cuidadosamente, y se cuantifica el contenido de LI mediante cromatografía líquida de alto rendimiento, alcanzando hasta el $94 \%$ de recuperación $\left(\left[\mathrm{C}_{2} \mathrm{mim}\right]\right.$ Cl) [43]. 
Otro método para la recuperación de líquidos iónicos de mezclas de hidrolizados de biomasa es mediante absorción, Binder y colaboradores demostraron por primera vez el uso de una resina de intercambio catiónico para la recuperación del LI de un producto de hidrólisis ácida mediante cromatografía líquida [39]. En esta técnica, una mezcla que contiene electrolitos y los solutos no electrolíticos se separan pasándose a través de una resina cargada. Las especies cargadas como el líquido iónico, son excluidas de la resina, mientras las no electrolíticas, como los azúcares son retenidas. En este estudio, la forma $\mathrm{H}^{+}$en la resina Dowex® 50WX4 fue en primer lugar completamente intercambiada por $\left[\mathrm{C}_{2} \mathrm{mim}\right]^{+}$. La resina intercambiada se utilizó como fase estacionaria en cromatografía líquida de exclusión iónica para recuperación de azúcares (glucosa y xilosa) y $\left[\mathrm{C}_{2} \mathrm{mim}\right][\mathrm{Cl}]$ de rastrojo de maíz hidrolizado. La glucosa, la xilosa y los LI se lograron recuperar hasta $94 \%, 86 \%$ y $92 \%$, respectivamente. Mai, Ahn y Koo implementaron este enfoque en la cromatografía de lecho móvil simulado de exclusión iónica (SMB) para la recuperación continua de glucosa, xilosa y [ mim $\mathrm{C}_{2}$ ] [OAc] del hidrolizado de biomasa acuosa con rendimientos de hasta 71.38, $99.37 \mathrm{y}$ 98.92\%, respectivamente [44]. Da Costa Lopes y Lukasik, efectuaron estudios de separación de xilosa y líquido iónico (emim) $\left(\mathrm{H}_{2} \mathrm{SO}_{4}\right)$, utilizando una columna cromatográfica y alúmina como fase estacionaria en presencia de acetonitrilo, optimizando la separación al utilizar una serie de variables, es decir, contenido de agua en el eluyente, tipo de tratamiento de alúmina y la concentración de la muestra utilizada, obteniendo los mejores resultados mediante el cambio de polaridad del sistema, alto contenido de acetonitrilo; además, el tratamiento de alúmina con $\mathrm{H}_{2} \mathrm{SO}_{4}$ permitió la recuperación cuantitativa de xilosa y LI, obteniéndose hasta el $90 \%$ en peso de los LI inicial en la primera fracción libre de xilosa [45].

\section{Conclusiones y recomendaciones}

Mediante esta revisión se puede concluir que la aplicación de líquidos iónicos como pretratamiento de la reacción de hidrólisis ácida para la obtención de monosacáridos en diferentes tipos de biomasa es efectiva, aumentando en la mayoría de los casos su rendimiento de reacción, siendo importante considerar el tipo de biomasa y las condiciones de disolución que son: relación másica LI/BM, temperatura y tiempo, además por sus características se considera que no emiten compuestos orgánicos volátiles potencialmente tóxicos, volviéndolos eficaces para los procedimientos sintéticos "verdes", que combinados con la utilización de biomasa residual contribuyen de manera positiva con el cuidado del medio ambiente. Debido a sus elevados costos es necesario para su aplicación a nivel industrial, considerar técnicas de recuperación y reciclaje de manera que los procesos se vuelvan rentables. Finalmente, se recomienda realizar estudios adicionales que permitan optimizar las condiciones de pretratamiento dependiendo de la composición estructural de la biomasa a utilizarse, del tipo de líquido iónico y las condiciones de disolución.

\section{Referencias}

[1] J. van Spronsen, M. A. T. Cardoso, G.J. Witkamp, W. de Jong, y M. C. Kroon, "Separation and recovery of the constituents from lignocellulosic biomass by using ionic liquids and acetic acid as co-solvents for mild hydrolysis", Chem. Eng. Process. Process Intensif., vol. 50, no. 2, p. 196-199, feb. 2011, doi: 10.1016/j.cep.2010.12.010.

[2] X. Yu et al., "Ultrasound-ionic liquid enhanced enzymatic and acid hydrolysis of biomass cellulose", Ultrason. Sonochem., vol. 41, p. 410-418, mar. 2018, doi: 10.1016/j.ultsonch.2017.09.003.

[3] A.-L. Li, X.-D. Hou, K.-P. Lin, X. Zhang, y M.-H. Fu, "Rice straw pretreatment using deep eutectic solvents with different constituents molar ratios: Biomass fractionation, polysaccharides enzymatic digestion and solvent reuse", J. Biosci. Bioeng., vol. 126, no. 3, p. 346-354, sep. 2018, doi: 10.1016/j.jbiosc.2018.03.011.

[4] Y. Zhang, G. Wei, G. Yu, y N. Qiao, "Hydrolysis of Straw in Ionic Liquids with Acid as Catalyst under Microwave Irradiation", $J$. Nanomater, vol. 2015, p. e709247, 2015, doi: 10.1155/2015/709247. 
[5] T. Vancov, A.-S. Alston, T. Brown, y S. McIntosh, "Use of ionic liquids in converting lignocellulosic material to biofuels", Renew. Energy, vol. 45, p. 1-6, sep. 2012, doi: 10.1016/j.renene.2012.02.033.

[6] [6] P. Reddy, "A critical review of ionic liquids for the pretreatment of lignocellulosic biomass", South Afr. J. Sci. Pretoria, vol. 111, no. 11/12, p. 1-9, 2015.

[7] S. K. Singh y A. W. Savoy, "Ionic liquids synthesis and applications: An overview", $J$. Mol. Liq., vol. 297, p. 112038, ene. 2020, doi: 10.1016/j.molliq.2019.112038.

[8] P. Bajpai, "Structure of Lignocellulosic Biomass", en Pretreatment of Lignocellulosic Biomass for Biofuel Production, P. Bajpai, Ed. Singapore: Springer, 2016, p. 7-12.

[9] V. Pasangulapati, K. D. Ramachandriya, A. Kumar, M. R. Wilkins, C. L. Jones, y R. L. Huhnke, "Effects of cellulose, hemicellulose and lignin on thermochemical conversion characteristics of the selected biomass", Bioresour. Technol, vol. 114, p. 663-669, jun. 2012, doi: 10.1016/j.biortech.2012.03.036.

[10] Y. Liao et al., "The role of pretreatment in the catalytic valorization of cellulose", Mol. Catal., vol. 487, p. 110883, may 2020, doi: 10.1016/j.mcat.2020.110883.

[11] C. Sievers, M. B. Valenzuela-Olarte, T. Marzialetti, I. Musin, P. K. Agrawal, y C. W. Jones, "Ionic-Liquid-Phase Hydrolysis of Pine Wood", Ind. Eng. Chem. Res., vol. 48, no. 3, p. 1277-1286, feb. 2009, doi: 10.1021/ie801174x.

[12] H. Tadesse y R. Luque, "Advances on biomass pretreatment using ionic liquids: An overview", Energy Environ. Sci., vol. 4, no. 10, p. 39133929, sep. 2011, doi: 10.1039/C0EE00667J.

[13] Li, Q. Wang, y Z. K. Zhao, "Acid in ionic liquid: An efficient system for hydrolysis of lignocellulose", Green Chem, vol. 10, no. 2, p. 177-182, 2008, doi: 10.1039/B711512A.
[14] S. Morales de la Rosa, "Hidrólisis ácida de celulosa y biomasa lignocelulósica asistida con líquidos iónicos”, 2015.

[15] Y.-L. Loow, T. Y. Wu, J. Md. Jahim, A. W. Mohammad, y W. H. Teoh, "Typical conversion of lignocellulosic biomass into reducing sugars using dilute acid hydrolysis and alkaline pretreatment", Cellulose, vol. 23, no. 3, p. 1491-1520, jun. 2016, doi: 10.1007/s10570-016-0936-8.

[16] Li, L. Sun, B. A. Simmons, y S. Singh, "Comparing the Recalcitrance of Eucalyptus, Pine, and Switchgrass Using Ionic Liquid and Dilute Acid Pretreatments", BioEnergy Res., vol. 6, no. 1, p. 14-23, mar. 2013, doi: 10.1007/s12155-012-9220-4.

[17] S. Sun, S. Sun, X. Cao, y R. Sun, “The role of pretreatment in improving the enzymatic hydrolysis of lignocellulosic materials", Bioresour. Technol., vol. 199, p. 49-58, ene. 2016, doi: 10.1016/j.biortech.2015.08.061.

[18] S.-H. Ho, S.-W. Huang, C.-Y. Chen, T. Hasunuma, A. Kondo, y J.-S. Chang, "Bioethanol production using carbohydrate-rich microalgae biomass as feedstock", Bioresour. Technol., vol. 135, p. 191-198, may 2013, doi: 10.1016/j.biortech.2012.10.015.

[19] L. Yan, A. A. Greenwood, A. Hossain, y B. Yang, "A comprehensive mechanistic kinetic model for dilute acid hydrolysis of switchgrass cellulose to glucose, 5-HMF and levulinic acid", $R S C A d v$, vol. 4, no. 45, p. 23492 , 2014, doi: 10.1039/c4ra01631a.

[20] L. Cao et al., "Optimizing xylose production from pinewood sawdust through dilute-phosphoric-acid hydrolysis by response surface methodology", J. Clean. Prod., vol. 178, p. 572-579, mar. 2018, doi: 10.1016/j.jclepro.2018.01.039.

[21] [21] E. Guerra-Rodríguez, O. M. PortillaRivera, L. Jarquín-Enríquez, J. A. Ramírez, y M. Vázquez, "Acid hydrolysis of wheat 
straw: A kinetic study", Biomass Bioenergy, vol. 36, p. 346-355, ene. 2012, doi: 10.1016/j.biombioe.2011.11.005.

[22] S. M. de la Rosa, "Hidrólisis ácida de celulosa y biomasa lignocelulósica asistida con líquidos iónicos", 2015.

[23] K. Świątek, S. Gaag, A. Klier, A. Kruse, J. Sauer, y D. Steinbach, "Acid Hydrolysis of Lignocellulosic Biomass: Sugars and Furfurals Formation", Catalysts, vol. 10, no. 4, Art. no. 4, abr. 2020, doi: 10.3390/catal10040437.

[24] Sun et al., "Production and extraction of sugars from switchgrass hydrolyzed in ionic liquids", Biotechnol. Biofuels, vol. 6, no. 1, p. 39, 2013, doi: 10.1186/1754-6834-6-39.

[25] D. Aboagye et al., "Glucose recovery from different corn stover fractions using dilute acid and alkaline pretreatment techniques", J. Ecol. Environ., vol. 41, no. 1, p. 26, jul. 2017, doi: 10.1186/s41610-017-0044-1.

[26] K. Dussan, D. Virginio da Silva, E. Moraes, P. Arruda, y M. FELIPE, "Dilute-acid hydrolysis of cellulose to glucose from sugarcane bagasse", Chem. Eng. Trans., vol. 38, p. 433, ene. 2014, doi: 10.3303/CET1438073.

[27] Y. P. Wijaya, R. D. D. Putra, V. T. Widyaya, J.-M. Ha, D. J. Suh, y C. S. Kim, "Comparative study on two-step concentrated acid hydrolysis for the extraction of sugars from lignocellulosic biomass", Bioresour. Technol., vol. 164, p. 221-231, jul. 2014, doi: 10.1016/j.biortech.2014.04.084.

[28] X. B. Lu, Y. M. Zhang, J. Yang, y Y. Liang, "Enzymatic Hydrolysis of Corn Stover after Pretreatment with Dilute Sulfuric Acid", Chem. Eng. Technol., vol. 30, no. 7, p. 938-944, 2007, doi: 10.1002/ceat.200700035.

[29] T.-C. Hsu, G.-L. Guo, W.-H. Chen, y W.-S. Hwang, "Effect of dilute acid pretreatment of rice straw on structural properties and enzymatic hydrolysis", Bioresour. Technol., vol. 101, no. 13, p. 4907-4913, jul. 2010, doi: 10.1016/j.biortech.2009.10.009.
[30] D. Greetham, J. M. Adams, y C. Du, "The utilization of seawater for the hydrolysis of macroalgae and subsequent bioethanol fermentation", Sci. Rep., vol. 10, no. 1, Art. no. 1, jun. 2020, doi: 10.1038/s41598-020-666109.

[31] W. Leenakul y N. Tippayawong, "Dilute acid pretreatment of bamboo for fermentable sugar production. Journal of Sustainable Energy \& Environment, 1(3), 117-120.”, 2010.

[32] I. Semerci y F. Güler, "Protic ionic liquids as effective agents for pretreatment of cotton stalks at high biomass loading", Ind. Crops Prod., vol. 125, p. 588-595, dic. 2018, doi: 10.1016/j.indcrop.2018.09.046.

[33] S. M. Sen, J. B. Binder, R. T. Raines, y C. T. Maravelias, "Conversion of biomass to sugars via ionic liquid hydrolysis: process synthesis and economic evaluation", Biofuels Bioprod. Biorefining, vol. 6, no. 4, p. 444-452, jul. 2012, doi: 10.1002/bbb.1336.

[34] T. G. Weldemhret et al., "Current advances in ionic liquid-based pre-treatment and depolymerization of macroalgal biomass", Renew. Energy, vol. 152, p. 283-299, jun. 2020, doi: 10.1016/j.renene.2020.01.054.

[35] L. T. P. Trinh, Y. J. Lee, J.-W. Lee, y H.-J. Lee, "Characterization of ionic liquid pretreatment and the bioconversion of pretreated mixed softwood biomass", Biomass Bioenergy, vol. 81, p. 1-8, oct. 2015, doi: 10.1016/j.biombioe.2015.05.005.

[36] R. Ratti, "Ionic Liquids: Synthesis and Applications in Catalysis", Adv. Chem., vol. 2014, p. 729842, oct. 2014, doi: 10.1155/2014/729842.

[37] N. Zhou, Y. Zhang, X. Gong, Q. Wang, y Y. Ma, "Ionic liquids-based hydrolysis of Chlorella biomass for fermentable sugars", Bioresour. Technol., vol. 118, p. 512-517, ago. 2012, doi: 10.1016/j.biortech.2012.05.074.

[38] S. Dee y A. T. Bell, "Effects of reaction conditions on the acid-catalyzed hydrolysis of 
miscanthus dissolved in an ionic liquid", Green Chem., vol. 13, no. 6, p. 1467-1475, jun. 2011, doi: 10.1039/C1GC15317J.

[39] J. B. Binder y R. T. Raines, "Fermentable sugars by chemical hydrolysis of biomass", Proc. Natl. Acad. Sci., vol. 107, no. 10, p. 4516-4521, mar. 2010, doi: 10.1073/pnas.0912073107.

[40] L. B. Malihan, G. M. Nisola, y W.-J. Chung, "Brown algae hydrolysis in 1-nbutyl-3-methylimidazolium chloride with mineral acid catalyst system", Bioresour. Technol., vol. 118, p. 545-552, ago. 2012, doi: 10.1016/j.biortech.2012.05.091.

[41] S. Kassaye, K. K. Pant, y S. Jain, "Hydrolysis of cellulosic bamboo biomass into reducing sugars via a combined alkaline solution and ionic liquid pretreament steps", Renew. Energy, vol. 104, p. 177-184, abr. 2017, doi: 10.1016/j.renene.2016.12.033.

[42] K. Shill, S. Padmanabhan, Q. Xin, J. M. Prausnitz, D. S. Clark, y H. W. Blanch,
"Ionic liquid pretreatment of cellulosic biomass: Enzymatic hydrolysis and ionic liquid recycle", Biotechnol. Bioeng., vol. 108, no. 3, p. 511-520, 2011, doi: 10.1002/bit.23014.

[43] Y. Gao, T. R. Bandara, y G. J. Griffin, "Acidcatalysed hydrolysis kinetics of sugar cane bagasse to glucose and xylose in selected ionic liquid media", IOP Conf. Ser. Earth Environ. Sci., vol. 307, p. 012005, ago. 2019, doi: 10.1088/1755-1315/307/1/012005.

[44] N. L. Mai, K. Ahn, y Y.-M. Koo, "Methods for recovery of ionic liquids-A review", Process Biochem., vol. 49, no. 5, p. 872-881, may 2014, doi: 10.1016/j.procbio.2014.01.016.

[45] A. M. da Costa Lopes y R. M. Łukasik, "Separation and Recovery of a HemicelluloseDerived Sugar Produced from the Hydrolysis of Biomass by an Acidic Ionic Liquid", ChemSusChem, vol. 11, no. 6, p. 1099-1107, mar. 2018, doi: 10.1002/cssc.201702231. 